BABELAO 5 (2016), p. 105-116

(C) ABELAO (Belgium)

\title{
L'édit de Milan entre tradition et renouveau
}

Par

\section{Pierre Maraval}

Université de Paris IV-Sorbonne

\section{Le contexte historico-politique de l'édit}

u mois de mars 313 , Constantin quitta Rome, dont il
s'était rendu maître lors de la fameuse bataille du Pont
Milvius, le 29 octobre de l'année précédente, bataille lors de laquelle celui qui régnait alors sur Rome, Maxence, s'était noyé dans le Tibre. Constantin se rendit alors à Milan pour y rencontrer Licinius et avoir des entretiens avec lui. Licinius était alors, avec Maximin Daïa en Orient, un des trois empereurs qui se partageaient le gouvernement de l'empire cette triarchie était le dernier état de la tétrarchie qu'avait inventée Dioclétien. Il est important de rappeler quels étaient leurs territoires respectifs : Constantin venait d'ajouter à son domaine primitif (Gaule, Espagne, Bretagne) l'Italie et l'Afrique, enlevées à Maxence; Licinius, à qui Galère avait attribué l'Italie et l'Afrique, mais s'était abstenu de les reprendre à Maxence, gouvernait de la Dalmatie à la Thrace, car Maximin Daïa, à la mort de Galère, s'était empressé de se rendre maître de l'Asie Mineure (une mesure peut-être décidée 
par Galère avant sa mort); Maximin, quant à lui, outre ce territoire, gouvernait la Syrie et l'Égypte.

Cette énumération de leurs territoires permet de constater d'emblée que Licinius, à cette date, est en position de faiblesse. Maximin Daïa a déjà étendu son domaine à ses dépens et Licinius a toutes les raisons de penser qu'il a l'intention de l'étendre davantage. Lui-même n'a pu ou voulu récupérer celui de Maxence, comme Galère le lui avait demandé. Constantin, en revanche, a agrandi son territoire. Il vient à cette rencontre auréolé de son succès au Pont Milvius, une victoire exceptionnelle, qui a été considérée par tous ses contemporains comme un don de Dieu. Constantin, en vérité, avait bien peu de chances de l'emporter: il avait entrepris la marche sur Rome contre l'avis de tous ses conseillers, ses troupes étaient moins nombreuses que celles de Maxence, elles arrivaient de Gaule après des semaines de marche, après le passage des Alpes, après de durs combats en Italie du nord qui en avaient déjà diminué le nombre. Rome, d'autre part, était à l'abri de ses murailles, un abri efficace, puisque dans les cinq années qui précédaient, elles avaient empêché Sévère, puis Galère, de s'en emparer; d'autre part, on était fin octobre, et Constantin pouvait craindre de devoir faire un long siège en hiver... Or Maxence, confiant dans un oracle qui annonçait la fin de l'ennemi des Romains (si l'on en croit Zosime ${ }^{1}$ ), fit l'erreur de sortir des remparts, ses troupes furent défaites en un seul jour; lui-même tomba dans le Tibre et se noya. Ce succès inattendu fut donc unanimement considéré comme le résultat d'une intervention divine. De nombreux récits, tant du côté païen que du côté chrétien, mirent en scène cette intervention, la mise en scène la plus connue étant celle de la vision dans le ciel la veille de la bataille - dont on peut pourtant rappeler que le premier témoin, Eusèbe de Césarée, ne la plaçait ni à Rome ni la veille la bataille, comme le feront ses successeurs, mais lors de « quelque expédition» non précisée ${ }^{2}$.

Donc, lors de cette rencontre, Constantin est en situation de force, d'autant plus que le sénat de Rome lui a attribué, après sa victoire, le titre de premier Auguste, que revendiquait Maximin Daïa depuis la mort de Galère (et qu'avaient reconnu Licinius

\footnotetext{
${ }^{1}$ ZosIME, Histoire nouvelle, II, 16, 1.

${ }^{2}$ Eusèbe, Vie de Constantin, I, 28, 2. La vision a lieu bien avant la descente sur Rome, et «l'armée qui le suivait dans quelque expédition » voit elle aussi le prodige. Socrate, $H E$, I, 2, 4 et Sozomène $H E$ I, 3, 3 en reste au « quelque part ». C'est Lactance qui en situant la vision (en songe) de Constantin juste avant la bataille, qui a fait placer la vision dans le ciel juste avant la bataille, fin octobre (Mort des persécuteurs [MDP], XLIV, 4). La Vie d'Alexandre et Métrophane (chez Photius, Bibliothèque, 216, 470 a) place l'apparition à l'époque de la bataille, après un premier engagement.
} 
et Constantin lui-même) ${ }^{3}$. Il a du reste déjà pris cette fonction au sérieux, car avant même de partir pour Milan, il a écrit à Maximin Daïa en lui ordonnant de faire cesser dans son territoire la persécution des chrétiens ${ }^{4}$, ce que Maximin avait refusé de faire après l'édit de tolérance de Galère du 30 avril 311 , et cette fois il a obtempéré (son édit de tolérance date de novembre ou décembre 312 , peu après la victoire de Constantin) ${ }^{5}$. Aussi, à mon sens, c'est Constantin qui inspirera les mesures prises à Milan, aussi bien les mesures de politique générale que celles qui concernent la politique religieuse.

Mesures de politique générale : les deux empereurs se sont « heureusement réunis, dira l'édit, "pour discuter de tous les problèmes relatifs à la sécurité et au bien public». Constantin vient à la rencontre de Licinius pour faire alliance avec lui (les deux y trouvent leur avantage) - ce qu'il concrétise en lui donnant sa sœur Constantia en mariage - et pour faire reconnaître à son allié les changements provoqués par sa victoire sur Maxence : son titre de premier Auguste, le fait que l'Italie et l'Afrique font maintenant partie de ses territoires, que Licinius n'a plus le droit de revendiquer. Il est clair aussi que cette alliance vise indirectement Maximin Daïa, ce qui explique que celui-ci ait obtempéré à l'ordre de Constantin de faire cesser la persécution, et que Licinius, que cette alliance renforce, accepte sans problème apparent les conditions posées par Constantin. Mais laissons ce point pour en revenir à notre sujet, l'édit de Milan, cet «édit » qui n'a peut-être jamais été un édit à proprement parler ou dont nous n'avons plus le texte, mais dont nous connaissons quelles mesures il décidait à la fois par la lettre que Licinius adressa à un gouverneur d'Asie mineure pour les faire appliquer (c'est le texte sans doute le plus proche de l'édit, s'il y en a eu un ${ }^{6}$ et par l'énoncé que fait Eusèbe de Césarée de ces constitutions impériales dans son Histoire ecclésiastique ${ }^{7}$. Comme l'a dit N. H. Baynes, «L'édit de Milan peut être une fiction, mais le fait que recouvre le terme reste entier ${ }^{8}$. D'autre part, bien qu'elles soient prises

\footnotetext{
${ }^{3}$ LACTANCE, MDP, XLIV, 11.

${ }^{4}$ Ibid., XXXVII, 1 ; EUSÈBE, Histoire ecclésiastique (HE), IX, 9, 12. Sur la date de ces litterae Constantini (que Lactance place trop tôt), voir Torben Christensen, C. Galerius Valerius Maximinus. Studies in the Politics and Religion in the Roman Empire AD 305-313, Ed. by Mogens Müller, Copenhague, 2012, p. 271-274. La nature, voire l'historicité de ces litterae, ne font pas l'unanimité parmi les historiens.

${ }^{5}$ EUSÈBE, $H E$, IXa, 1, 2-6.

${ }^{6}$ LACTANCE, MDP, XLVIII.

${ }^{7}$ EusÈBE, $H E, X, 5,4-5$.

8 N.H. BAYNES, Constantine the Great and the Christian Church, Londres, 1929, réimp. 1972, p. 11.
} 
par les deux empereurs, ces mesures sont semblables à celles que Constantin avait déjà fait appliquer en Afrique peu après son arrivée à Rome $^{9}$, ce qui contredit ceux qui ont voulu en attribuer la paternité à Licinius; à mon sens, la lettre de Licinius, si on la compare aux autres lettres de l'époque de Constantin, c'est du Constantin.

\section{Le caractère traditionnel de l'édit : obtenir la faveur divine}

L'édit de Milan a un caractère traditionnel : comme l'édit de tolérance de Galère d'avril 311, qu'il complète (et dont on pourrait considérer, à première vue, qu'il constitue les décrets d'application - d'autant que Constantin faisait partie des signataires de cet édit, bien qu'il n'y fût sans doute pour rien) ${ }^{10}$, comme le rescrit que Maximin adresse à ses sujets d'Orient en avril 312, qui eut pour effet de prolonger la persécution, il considère que le bonheur de l'État est procuré par la faveur divine et obtenu par le culte rendu à Dieu par tous. "Que tous se réjouissent de ce que, grâce à Notre Piété, à nos cérémonies sacrées, à l'honneur rendu aux dieux, la force très puissante et très ferme de l'air s'est adoucie ${ }^{11}$, et il rend responsable des maux récents l'attitude des chrétiens, qui ne prient pas les dieux. Galère, de son côté, constate qu'un des fâcheux résultats des édits de persécution, c'est que les chrétiens «ni n'accordaient aux dieux célestes le culte qui leur est dû, ni n'honoraient le Dieu des chrétiens $»^{12}$. Il leur demande donc, en retour de la tolérance qu'il leur accorde, de «prier leur Dieu pour notre salut, celui de l'État et le leur, afin que de les affaires publiques soient de toute manière en bon état ${ }^{13}$. Le motif est politique : il est dangereux pour l'empire romain que les chrétiens ne prient plus aucun dieu ${ }^{14}$ ! L'édit de Milan tient le même discours : parmi les problèmes relatifs à la sécurité et au bien public, il a mis au premier rang « ceux qui assurent le bien de la majorité, ceux sur lesquelles repose le respect de la divinité, afin que tout ce qu'il y a de divin au céleste séjour nous soit favorable et propice, à nous-mêmes et à tous ceux qui se trouvent sous notre autorité ». Ainsi, dans les deux édits, s'exprime une conception traditionnelle de la religion, dans

\footnotetext{
${ }^{9}$ Constantin, Lettre 1 (fin 212, début 213). Les lettres sont citées avec leur numérotation dans Constantin, Lettres et discours, trad. P. Maraval, Paris 2010.

10 Eusèbe, HE, VIII, 17, 4. Lactance, MDP, XXXIV, a omis le préambule de la loi, qui comportait la titulature des empereurs.

${ }^{11}$ EusÈBE, HE, IX, 7, 11.

${ }^{12}$ Ibid., VIII, 17, 9.

${ }^{13}$ Ibid., 10.

${ }^{14}$ T. CHRISTENSEN, op. cit., p. 175.
} 
laquelle la piété envers les dieux assure en retour la stabilité, la paix et la prospérité de l'empire. De ce point de vue, il n'est pas faux d'écrire que «Constantin ne fait qu'adhérer tout simplement à l'antique précepte du do ut des de la religion païenne ${ }^{15}$. Les deux édits s'inspirent cependant de considérations bien différentes, et la conclusion qu'ils en tirent l'est tout autant. L'édit de Galère était un aveu d'échec: la persécution n'avait pas éradiqué le christianisme, les chrétiens n'étaient pas revenus « au bon sens », ils « demeuraient dans la même folie »; malgré cela, l'empereur, par pure philanthropie (vertu impériale), leur accordait son pardon et les autorisait à exister, tout en leur demandant, dans sa grande clémence, de prier Dieu pour son salut. En revanche, les mesures prises à Milan, tout en ayant pour but, elles aussi, «la sécurité, la prospérité publique ", n'entendent pas corriger une politique religieuse qui avait échoué : elles déclarent au contraire vouloir prolonger la faveur divine dont les empereurs venaient "d'éprouver les effets dans des circonstances si graves "- ce qui est une claire allusion à la victoire de Constantin, et seulement à celle-ci.

Or comment ces mesures prolongent-elles la faveur divine ? Tout d'abord en instaurant une politique de consensus en matière de religion, en accordant à chacun « la libre faculté de pratiquer le culte de son choix ». Après des années où la persécution avait provoqué une véritable discorde civile entre les adeptes des cultes anciens et les chrétiens, ces mesures entendaient réconcilier les uns et les autres, en donnant aux chrétiens une place reconnue dans l'empire, tout en laissant toute liberté à la religion traditionnelle : «La même possibilité d'observer leur religion et leur culte est concédée à tous les citoyens, ouvertement et librement». Les signataires, Constantin et Licinius, déclarent qu'ils cherchent, " avant tout, à assurer le respect et l'honneur de la divinité », et c'est pourquoi il donnent, aux chrétiens comme à tous, " la liberté et la possibilité de suivre la religion de leur choix ». L'édit de Milan est donc d'abord un édit de tolérance religieuse de portée générale, qui spécifie que celle-ci concerne aussi les chrétiens. Les dispositions précédentes, celles des persécuteurs, sont déclarées «tout à fait malencontreuses et étrangères à notre mansuétude ». Est donc récusé tout recours à la violence, implicitement affirmé que la religion ne doit pas être contrainte, ce qui tranche avec les édits de la grande persécution. "Moins tranchant et conflictuel que Dioclétien, qui exclut de l'empire chrétiens et manichéens, Constantin fut loin d'élever le christianisme en religion d'État et de frapper d'interdiction les

${ }^{15}$ D. De DECKER, Le De mortibus persecutorum : un libelle au service de la propagande constantinienne, Iaşi, 2013, p. 409. 
cultes traditionnels $»{ }^{16}$. Ce n'est pas lui que l'on peut accuser d'être à l'origine d'une politique d'intolérance ${ }^{17}$.

\section{La nouveauté: la faveur est attendue de la divinité suprême}

D'autre part, si les deux édits traduisent la même conviction sur le lien entre bonheur de l'empire et pratique religieuse, l'édit de Milan introduit une autre nouveauté : la bienveillance divine n'est plus attendue des dieux immortels, comme le disaient Galère ou Maximin Daïa dans leurs édits, mais de « la divinité suprême » (summa divinitas) (Lactance), à laquelle les signataires déclarent rendre hommage. Une lettre adressée à la même époque par Constantin au proconsul païen d'Afrique le redit de la manière la plus claire : «Il est manifeste, à partir d'un grand nombre de faits, que la négligence de la religion, dans laquelle est conservé le respect souverain de la très sainte et très haute <puissance>, est cause de grands dangers pour les affaires de l'État; en revanche, lorsque la religion est reçue et gardée conformément aux lois, elle apporte, procurées par les bienfaits divins, une très grande prospérité au nom romain et une réussite notable à toutes les entreprises humaines ${ }^{18}$. C'est donc désormais le Dieu suprême, la très haute puissance, et non plus les dieux de la religion traditionnelle, qui assure la protection de l'empire. L'édit de Milan est monothéiste, à tout le moins monothéisant, d'une sorte de «monothéisme neutre ${ }^{19}$, qui tolère les différences d'approche, mais est nettement affirmé. De surcroît, en réservant le recours à la divinité suprême, à "tout ce qu'il y a de divin au céleste séjour » (Lactance), « tout ce qu'il peut y avoir de divinité et de puissance céleste » (Eusèbe), il exclut les divinités d'en bas, autrement dit le polythéisme local et populaire de la superstition $^{20}$.

On a ici un changement de perspective important : la liberté totale est accordée à toute religion, mais ne méritent

16 Polymnia ATHANASSIADI, Vers la pensée unique. La montée de l'intolérance dans l'Antiquité tardive, Paris 2010, p. 58.

17 Voir H. A. Drake, Constantine and the Bishops. The Politics of Intolerance, Baltimore et Londres, 2000, dont un des premiers recenseurs (S.G. Hall, JTS 52, 2001, p. 895) remarquait que l'A. aurait aussi bien pu lui donner comme sous-titre, s'agissant de Constantin (et non des évêques), « la politique de tolérance». Ce sont les luttes entre chrétiens qui ont conduit à l'utilisation par les évêques des moyens coercitifs de l'État en faveur de leurs croyances.

${ }^{18}$ CONSTANTIN, Lettre 3, 1 (avant avril 313).

${ }^{19}$ L'expression est de Charles PIETRI, Histoire du christianisme, II, Paris 19 , p. 201.

${ }^{20}$ R. TURCAN, Constantin en son temps. Le baptême ou la pourpre? Paris, 2006, p. 174. 
l'appellation de religion que celles qui rendent un culte à la divinité suprême, dont pourtant l'identité n'est pas précisée. Si, pour Constantin, il s'agit déjà du Dieu chrétien, il n'en est pas de même pour Licinius, qui restera païen, mais païen monothéisant : deux ans plus tard, avant sa bataille définitive contre Maximin Daïa, si l'on peut se fier à Lactance sur ce point, il fera réciter à son armée une prière au Dieu suprême (dont certains historiens supposent, peut-être à juste titre, qu'elle avait été composée par Constantin) ${ }^{21}$ - ce qui ne l'empêchera pas, lors de son conflit ultérieur avec Constantin, de s'en prendre aux chrétiens de son territoire, tenus non sans raison pour une cinquième colonne favorable à son rival. Une des conséquences de cette définition de la religion est que pour la première fois, dans l'empire, le christianisme est explicitement reconnu comme religio, alors que jusque-là il était considéré comme une superstition. Souvenons-nous des termes qu'employaient Tacite, Suétone, Pline le Jeune, " superstition exécrable ", "superstition nouvelle et maléfique », «superstition déraisonnable et sans mesure » ${ }^{22}$; l'édit de Galère parlait de folie, Maximin d'erreur aveugle, d'exécrable folie $^{23}$. Or le texte parle de la «religion des chrétiens » (le grec utilise $\pi \alpha \rho \alpha \varphi v v ́ \lambda \alpha \xi 1 \zeta$, observance, et $\theta \rho \eta \sigma \kappa \varepsilon i ́ \alpha$, le latin observantia et religio), alors qu'à l'inverse il considère implicitement celles qui s'adressent aux divinités inférieures comme des pratiques superstitieuses. Ce ne sera pas sans conséquence pour la politique ultérieure de Constantin.

\section{Mesures ponctuelles en faveur des chrétiens}

Pour l'instant cependant, l'édit se contente d'ajouter à la mesure essentielle, la liberté pleine et entière de religion et de culte (toujours liées chez les Romains, où il n'y a pas de religion sans pratique du culte), une série de mesures ponctuelles en faveur des chrétiens, qui sont d'abord des mesures de justice : il est décidé que seront rendus aux chrétiens les bâtiments qui leur ont été confisqués lors de la persécution, essentiellement les lieux où ils avaient l'habitude de se réunir. Ils devront même leur être rendus immédiatement, et ceux qui les avaient reçus en donation de l'État sont tenus de les rendre aussitôt - un dédommagement toutefois pourra leur être accordé ! Ces mesures constituent un progrès par rapport à l'édit de Galère, qui se contentait d'autoriser les chrétiens à

${ }^{21}$ Lactance, MDP, XLVI, 6 ; A. Piganiol, Mélanges H. Grégoire (= Byzantion, 35, 1-2), 1965, p. 515. La prière est très proche de celle que Constantin prescrit de faire réciter à son armée le jour du soleil : Eusèbe, Vie de Const., IV, 20.

${ }^{22}$ TACITE, Annales, XV, 44, 3 ; SuÉtone, Vie des Douze Césars. Néron, 16 ; Pline LE JeUne, Lettres, X, 96, 7.

${ }^{23}$ EusÈBE, $H E$ VIII, 17, 9 ; IX, 7, 11-12. 
reconstruire les églises qui, conformément au premier édit de persécution, avaient été détruites par les autorités. Elles témoignent déjà de la bienveillance de Constantin, une bienveillance qui s'est déjà exercée à Rome avant même la rencontre de Milan et qui ne cessera de s'accentuer.

Il est certain en effet que, pour Constantin, l'édit de Milan n'était qu'un minimum, un point de départ, alors que pour Licinius c'était un maximum. Il sera suivi en effet d'une politique favorable aux chrétiens et à l'Église (car Constantin a déjà le sens de l'institution, qu'il saura utiliser à son profit). A l'Église en tant que collège reconnu, Constantin accorda des dons - d'argent ou de terrains, permettant ainsi la construction de vastes basiliques, à Rome, à Jérusalem et ailleurs -, l'autorisation de recevoir des héritages, l'exemption de l'impôt foncier. Ces mesures devaient provoquer un développement considérable des biens de l'Église, à laquelle toutefois il était demandé d'assurer une fonction de sécurité sociale dont l'État ne se chargeait pas. Les clercs reçurent aussi quelques privilèges fiscaux, ceux dont bénéficiaient déjà les prêtres païens ainsi que certaines professions, signe simplement que leur fonction était maintenant reconnue. Parmi ces privilèges, la loi qui donne aux évêques un droit d'arbitrage dans les procès au civil constituait en fait, pour les évêques, une lourde charge non rétribuée. Tout cela fut de grande conséquence: en donnant à l'Église une place reconnue, et sur certains points privilégiée, Constantin a favorisé sa visibilité et son développement ${ }^{24}$.

\section{Le christianisme, seule véritable religio}

De surcroît, à mesure que le temps de son règne avançait, Constantin allait affirmer de plus en plus ouvertement son choix du christianisme. En 324, après avoir vaincu Licinius et être devenu le maître de tout l'empire, il adressa aux païens et aux chrétiens d'Orient qui venaient de passer sous son autorité deux lettres qui montrent non seulement ce choix, mais sa conviction que les chrétiens, dont il fait partie, possèdent « la demeure resplendissante de la vérité $»$; ce qu'il exprime en s'adressant à Dieu (la demeure... " que tu nous as donnée ») et en lui demandant que cette possession soit donnée aussi aux païens $^{25}$. Ces lettres, qui sont des lettres officielles (la première se termine par «à publier dans nos régions orientales ») ${ }^{26}$, auxquelles on peut ajouter le Discours à l'assemblée des saints,

\footnotetext{
${ }^{24}$ Sur les faveurs accordées à l' Église et aux clercs, voir P. MARAVAL, Constantin le Grand, empereur romain, empereur chrétien, Paris, 2011, p. 114-119.

${ }^{25}$ Constantin, Lettre $15,14$.

${ }^{26}$ ID, Lettre 13, 31.
} 
prononcé devant sa cour un jour de $325^{27}$, peu avant le concile de Nicée, ont un ton et une portée qui vont au-delà de ceux de l'édit de Milan. Ces textes montrent, en particulier, que la conception que se fait Constantin de la religion a évolué : ce terme, pour lui, désigne désormais le seul christianisme, la vraie religion. S'il y continue d'accorder aux païens la liberté de culte, c'est par seul souci de la paix et du bien commun. Ils peuvent, s'ils le veulent, «fréquenter les temples du mensonge ", mais Constantin leur dit clairement qu'ils sont " dans l'erreur ${ }^{28}$, que leur culte est superstition $(\delta \varepsilon \imath \sigma ı \delta \alpha \mu o v i ́ \alpha)^{29}$. Avec ces textes, le christianisme est passé de la périphérie de la superstition privée au centre de la religion publique. Constantin a sans doute lu à cette époque les Institutions Divines du maître de rhétorique de son fils Crispus, Lactance, qui lui avait dédié la deuxième édition de son ouvrage $^{30}$. Il en partage du moins le point de vue. Lactance écrivait: «La religion est du domaine du vrai culte, la superstition du faux ; aussi les adorateurs des dieux se croient religiosi, alors qu'ils sont superstitiosi ${ }^{31}$, car seul le christianisme est religio $»^{32}$. En écho, Constantin, dans ses lettres, l'appelle " la religion véritable et pure », " la religion pure et authentique qui procède de l'accord sur le divin ${ }^{33}$. Malgré cela, il n'entend pas contraindre les païens à adopter le christianisme. Lactance invitait lui aussi à la discussion argumentée avec les intellectuels païens et les prêtres de la religion païenne, car nul ne doit être délivré de sa folie, de sa sottise (amentia, stultitia) contre son gré ${ }^{34}$; les chrétiens sont donc invités à la «tolerantia malorum », qui n'est pas ce que nous appelons la tolérance, mais est une attitude de patience, qui supporte des maux qu'elle ne peut corriger ${ }^{35}$. Constantin, s'adressant à des chrétiens, prône une attitude semblable : « Si quelqu'un fait obstacle à sa guérison, qu'il n'en soit pas blâmé par un autre, car la guérison des maladies est proposée à tous, est sous les yeux de tous... Ce que chacun a vu et compris,

${ }^{27}$ Sur la date (discutée) de ce discours, voir l'introduction à Constantin, Lettres et discours, p. XXII.

${ }^{28}$ ID., Lettre 15,13 et 14.

${ }^{29}$ ID., Lettre 27, 2 ; Discours à l'assemblée des saints, I, 5 ; XVI, 1 ; XVIII, 2.

${ }^{30}$ LACTANCE, Institutions divines, I, 1, 13-15.

${ }^{31}$ Ibid., IV, 28, 11.

${ }^{32}$ Ibid., V, 4, 8 .

${ }^{33}$ COnSTANTIn, Lettre 28, 5 ; Lettre 40, 7.

${ }^{34}$ ID., Discours à l'assemblée des saints, V, 19, 10-13.

${ }^{35}$ LACTANCE, Institutions divines, V 7, 5 s. ; 22, 2 s. ; VI, 18, 18 s., 29 ; VII, 5,15 . 
qu'il l'utilise, si possible, pour aider autrui, mais si c'est impossible, qu'il s'abstienne. C'est une chose d'accueillir librement le combat pour l'immortalité, c'en est une autre de l'imposer avec des sanctions $»^{36}$.

Cette attitude de tolérance, de patience envers les erreurs des païens s'est maintenue durant tout le règne de Constantin, mais elle ne l'a pas empêché de tenter d'interdire tout ce que, dans la religion traditionnelle, il considérait comme pratique superstitieuse : il a voulu, comme le disait Alföldi, «désinfecter » ${ }^{37}$, purifier le paganisme. D'où, bientôt, l'interdiction de l'haruspicine (la divination à partir de l'examen des entrailles des victimes), celle des pratiques magiques, et finalement celle des sacrifices sanglants ${ }^{38}$, qu'il tolérait encore, quoique de manière particulièrement méprisante, dans son Discours à l'assemblée des saints : "Allez-vous en donc, impies - cela vous est permis parce que votre péché est incorrigible -, allez aux égorgements des victimes sacrées, aux festins, aux fêtes, aux beuveries, en prétendant accomplir un culte alors qu'en réalité vous vous adonnez à l'intempérance et aux plaisirs $»{ }^{39}$. On voit ici que la liberté accordée n'est pas celle, positive, que l'édit de Milan reconnaissait aux chrétiens : c'est la tolérance du moindre mal, celle que Constantin accorda aussi aux donatistes, par découragement devant leur obstination. L'interdiction des sacrifices sanglants, du reste, ne sera pas respectée, comme le montre sa constante répétition chez ses successeurs jusqu'à Théodose et même au-delà, et Constantin, par sagesse politique, dans un empire qui était encore majoritairement païen, ne chercha pas vraiment à la faire appliquer exigeant toutefois que le temple bâti en l'honneur de sa famille à Hispellum ne soit "souillé par les fraudes d'aucune superstition contagieuse ${ }^{40}$, donc à tout le moins par les sacrifices sanglants. Mais on sait aussi qu'il conserva le titre de Pontifex maximus (Souverain Pontife) de la religion païenne et les attributions de cette charge, qui lui donnait autorité sur le calendrier civil et religieux et sur le recrutement des collèges sacerdotaux païens, qu'il laissa subsister toutes les institutions religieuses existantes, les temples (aucun ne fut détruit à Rome), les collèges des pontifes, ceux des vestales; la statue de

${ }^{36}$ CONSTANTIN, Lettre 15, 19.

${ }^{37}$ A. ALFÖLDI, The Conversion of Constantine Constantine and Pagan Rome, Oxford, ${ }^{2} 1969$, p. 106 (sterilized).

38 Eusèbe, Vita Constantini, II, 45; Cod. Theod., XVI, 10, 1 (haruspicine) ; XVI, 10, 2 (sacrifices : loi de Constance et Constant de 341, mais qui en appelle à une loi de leur père non retenue dans le Code). Sur cette question débattue, voir P. MARAVAL, op. cit., p. 266-268.

${ }^{39}$ Constantin, Discours à l'assemblée des saints, XI, 7.

${ }^{40}$ CIL XI, 5265. 
la Victoire resta au Sénat, les subsides publics pour les anciens cultes continuèrent d'être versés. Il restait fidèle en cela au refus de la contrainte, des «stipulations tout à fait malencontreuses » des persécuteurs qu'il dénonçait dans l'édit de Milan, et mieux encore au principe qu'il posait dans une de ses lettres de 324: "Je désire, pour le bien commun de l'univers et de tous les hommes, que ton peuple soit en paix et reste exempt de troubles. Que ceux qui sont dans l'erreur, joyeux, reçoivent la jouissance de la même paix et de la même tranquillité que les croyants ", - et il donne la raison de cette attitude dans cette phrase magnifique, mais sans doute bien utopique - « car la douceur de la concorde aura de la force pour les corriger eux aussi et les conduire dans le droit chemin ». Donc, «que personne n'en moleste un autre, que chacun retienne et pratique ce que désire son âme ». L'édit de Milan parlait déjà, à propos du christianisme, "d'adhésion réfléchie ", de «libre détermination», et demandait de respecter cette attitude. Il n'en reste pas moins que Constantin, en 324, affirme nettement sa préférence : "Mais il faut que ceux qui ont de saines pensées soient convaincus que seuls vivront de manière sainte et pure ceux que tu appelles à se reposer sur tes saintes lois ${ }^{41}$. Les bons citoyens, aux yeux de l'empereur, ce sont désormais les chrétiens, les seuls à bien se conduire.

\section{Constantin didascale}

Ces lettres de 324 mettent aussi clairement en évidence le Constantin enseignant, didascale, celui qui s'était convaincu que son devoir était de diffuser le christianisme. Conduire ses sujets à la connaissance de Dieu était du reste une des tâches assignées aux souverains de l'époque hellénistique. Aussi l'empereur chrétien sera largement incité à être un héraut de la foi par son entourage d'évêques, en particulier par Eusèbe de Césarée, qui dans son discours pour les trente ans de règne, en juillet 336, lors des tricennalia soluta, lui rappelait ses devoirs, fondés sur l'origine divine de son pouvoir et sur l'imitation de son modèle, le Logos: "Comme un interprète du Verbe de Dieu, (l'empereur) appelle tout le genre humain à la connaissance du Tout-Puissant, en criant d'une voix forte aux oreilles de tous et en proclamant les lois de la piété véritable à tous les habitants de la terre... Dans son zèle pour le ToutPuissant, ayant purifié de toute souillure d'erreur athée le royaume terrestre, il convoque le chœur des saints et des hommes pieux à l'intérieur des demeures royales, en veillant à assurer le salut commun de la flotte tout entière de ceux dont il est le pilote ${ }^{42}$. Constantin prendra cette tâche à cœur. Dès 315, il écrivait à un gouverneur africain : «Que dois-je faire de

\footnotetext{
${ }^{41}$ Constantin, Lettre 15, 13.

${ }^{42}$ EusÈBE, Triakontaétérikos (Louanges de Constantin), II, 4-5.
} 
plus, du fait de ma fonction et ma charge de prince, une fois dissipées les erreurs et détruites toutes les témérités, sinon présenter à tous la vraie religion, une concorde loyale et le culte dû au Dieu tout-puissant? » ${ }^{43}$. Et en 335, il devait écrire aux évêques réunis en concile à Tyr: "Même les barbares aujourd'hui, grâce à moi, l'authentique serviteur de Dieu, ont reconnu Dieu et ont appris à le louer $\gg{ }^{44}$. L'édit de Milan, aussi bien en ce qu'il avait de traditionnel qu'en ce qu'il avait de nouveau, contenait cela en germe.

${ }^{43}$ Constantin, Lettre 10, 5 (315).

${ }^{44}$ Constantin, Lettre 37, 10 (335). 